\title{
THE INVERSION OF HANKEL TRANSFORMS OF ORDER ZERO AND UNITY
}

\author{
by IAN N. SNEDDON
}

(Received 2 September, 1968)

1. Introduction. In teaching the elements of transform theory to students of physics and engineering it is very useful to have available, as early as possible, the inversion theorem for the Hankel transform

$$
f_{v}(\xi) \equiv \mathscr{H}_{v}[f(x) ; \xi]=\int_{0}^{\infty} x f(x) J_{v}(\xi x) d x .
$$

The difficulty is that a valid proof for general values of $v$ (cf. [1], p. 456) is complicated and involves a greater familiarity with the processes of analysis and the properties of Bessel functions than is possessed by most science students.

In applications of the Hankel transform to the solution of boundary value problems in mathematical physics (e.g. in potential theory and in the theory of elasticity) it is usually only the transforms of order zero and unity that are involved. The object of the present note is to show that a convincing demonstration of the result $\mathscr{H}_{v}^{-1}=\mathscr{H}_{v}$ in these cases can be given to students who are familiar with the Fourier inversion theorem, with the fact that the solution of the Abel integral equation

is

$$
\int_{0}^{x} \frac{f(t) d t}{\sqrt{\left(x^{2}-t^{2}\right)}}=g(x) \quad(x>0)
$$

and with the results

$$
f(t)=\frac{2}{\pi} \frac{d}{d t} \int_{0}^{t} \frac{x g(x) d x}{\sqrt{\left(t^{2}-x^{2}\right)}} \quad(t>0)
$$

$$
\begin{aligned}
& \int_{0}^{x} \frac{\cos (\xi t)}{\sqrt{\left(x^{2}-t^{2}\right)}} d t=\frac{1}{2} \pi J_{0}(\xi x) \quad(x>0, \xi>0), \\
& \int_{x}^{\infty} \frac{\sin (\xi t) d t}{\sqrt{\left(t^{2}-x^{2}\right)}}=\frac{1}{2} \pi J_{0}(\xi x) \quad(x>0, \xi>0) .
\end{aligned}
$$

It should also be observed that a simple change of variables in equations (1.2) and (1.3) shows that the solution of the integral equation

is

$$
\int_{x}^{\infty} \frac{f(t) d t}{\sqrt{\left(t^{2}-x^{2}\right)}}=g(x) \quad(x>0)
$$

$$
f(t)=-\frac{2}{\pi} \frac{d}{d t} \int_{t}^{\infty} \frac{x g(x) d x}{\sqrt{\left(x^{2}-t^{2}\right)}} .
$$


All of this should be known (quite early on) to students attending a " mathematical methods " course.

The proof that in the cases $v=0,1, \mathscr{H}_{v}^{-1}=\mathscr{H}_{v}$, i.e. that the integral equation (1.1) has solution

$$
f(x)=\int_{0}^{\infty} \xi f_{v}(\xi) J_{v}(\xi x) d \xi
$$

rests on interpreting the solution (1.2) as an inversion theorem for an operator $\mathscr{A}_{1}$ defined (apart from a numerical factor) by equation (1.2) called, for obvious reasons, an Abel transform, and the solution (1.7) as an inversion theorem for an operator $\mathscr{A}_{2}$ whose definition is suggested by equation (1.6).

The basic identities for these operators are equations (2.13) and (2.14) below. By taking simple forms for the arbitrary kernel $K(\xi, x)$ occurring in these equations we can immediately derive the desired inversion theorems for the Hankel transform (cf. Section 3). In Section 4 it is shown that the same method can be applied to derive the inversion theorem for H-transforms (see [2], p. 215) in the case $v=0$.

2. Abel transforms. It is convenient to define "Abel transforms " $\mathscr{A}_{1}$ and $\mathscr{A}_{2}$ through the equations

$$
\begin{aligned}
& \hat{f}_{1}(x) \equiv \mathscr{A}_{1}[f(t) ; x]=\int\left(\frac{2}{\pi}\right) \int_{0}^{x} \frac{f(t) d t}{\sqrt{\left(x^{2}-t^{2}\right)}}, \\
& \hat{f}_{2}(x) \equiv \mathscr{A}_{2}[f(t) ; x]=\sqrt{ }\left(\frac{2}{\pi}\right) \int_{x}^{\infty} \frac{f(t) d t}{\sqrt{\left(t^{2}-x^{2}\right)}} .
\end{aligned}
$$

If we regard equation (2.1) as an integral equation for $f(t), \hat{f}_{1}(x)$ being prescribed, then its solution is given by equation (1.2). Making use of the notation

$$
\hat{f}_{1}=\mathscr{A}_{1} f \Rightarrow f=\mathscr{A}_{1}^{-1} \hat{f}_{1},
$$

we see that this solution can be written in the operator form

$$
\mathscr{A}_{1}^{-1} \hat{f}_{1}=D_{t} \mathscr{A}_{1}\left[x \hat{f}_{1}(x) ; t\right],
$$

where, as usual, $D_{t}$ denotes the differential operator $d / d t$.

Similarly, if we regard (2.2) as an integral equation for $f(t)$ we find that

$$
\mathscr{A}_{1}^{-1} \hat{f}_{2}=-D_{t} \mathscr{A}_{2}\left[x \hat{f}_{2}(x) ; t\right] \text {. }
$$

Many standard integrals can be interpreted as statements involving these transforms. For instance, we can interpret the integrals (1.3) and (1.4) in the forms

$$
\begin{aligned}
& \mathscr{A}_{1}[\cos (\xi t) ; x]=\sqrt{ }\left(\frac{1}{2} \pi\right) J_{0}(\xi x), \\
& \mathscr{A}_{2}[\sin (\xi t) ; x]=\sqrt{ }\left(\frac{1}{2} \pi\right) J_{0}(\xi x),
\end{aligned}
$$


respectively. If we differentiate both sides of (2.6) with respect to $\xi$ we obtain the formula

$$
\mathscr{A}_{1}[t \sin (\xi t) ; x]=\sqrt{ }\left(\frac{1}{2} \pi\right) x J_{1}(\xi x),
$$

and using this result in equation (2.4) we find that

$$
\mathscr{A}_{1}^{-1}[\sin (\xi x) ; t]=\sqrt{ }\left(\frac{1}{2} \pi\right) \xi t J_{0}(\xi t) \text {. }
$$

Similarly from equations (2.5) and (2.7) we find that

$$
\mathscr{A}_{2}^{-1}\left[\frac{\sin (\xi x)}{x} ; t\right]=-\frac{d}{d t} \mathscr{A}_{2}[\sin (\xi x) ; t]=-\sqrt{ }\left(\frac{1}{2} \pi\right) \frac{d}{d t} J_{0}(\xi t)
$$

from which we deduce immediately the equation

$$
\mathscr{A}_{2}^{-1}\left[\frac{\sin \xi x}{x} ; t\right]=\sqrt{ }\left(\frac{1}{2} \pi\right) \xi J_{1}(\xi t)
$$

We have similar results for the Struve function $\mathrm{H}_{0}(\xi x)$ and the Bessel function of the second kind of order zero, $Y_{0}(\xi x)$. If we replace the function $\sin (\xi t)$ by its Maclaurin expansion and integrate term by term, we can easily show that

$$
\mathscr{A}_{1}[\sin (\xi t) ; x]=\sqrt{ }\left(\frac{1}{2} \pi\right) \sum_{r=0}^{\infty} \frac{(-1)^{r}\left(\frac{1}{2} \xi x\right)^{2 r+1}}{\Gamma\left(r+\frac{3}{2}\right) \Gamma\left(r+\frac{3}{2}\right)} .
$$

Now Struve's function $\mathbf{H}_{v}(x)$ is defined by the equation

$$
\mathbf{H}_{v}(x)=\sum_{r=0}^{\infty} \frac{(-1)^{r}\left(\frac{1}{2} x\right)^{v+2 r+1}}{\Gamma\left(r+\frac{3}{2}\right) \Gamma\left(v+r+\frac{3}{2}\right)}
$$

so that the above equation is equivalent to the relation

$$
\mathscr{A}_{1}[\sin (\xi t) ; x]=\sqrt{ }\left(\frac{1}{2} \pi\right) \mathbf{H}_{0}(\xi x) \text {. }
$$

On the other hand, if we define the Bessel function of the second kind by its integral representation

$$
Y_{0}(x)=-\frac{2}{\pi} \int_{1}^{\infty} \frac{\cos (x t) d t}{\sqrt{\left(t^{2}-1\right)}}
$$

(cf. [1], p. 170), we see immediately that

$$
\mathscr{A}_{2}[\cos (\xi t) ; x]=-\sqrt{ }\left(\frac{1}{2} \pi\right) Y_{0}(\xi x) .
$$

If we differentiate both sides of this equation with respect to $\xi$, interchange $x$ and $t$ and use the recurrence relation $Y_{0}^{\prime}=-Y_{1}$, we find that

$$
\mathscr{A}_{2}[x \sin (\xi x) ; t]=-\sqrt{ }\left(\frac{1}{2} \pi\right) t Y_{1}(\xi t) .
$$

Now making use of the formula (2.5) and the recurrence relation

$$
\frac{d}{d z}\left\{z Y_{1}(z)\right\}=z Y_{0}(z)
$$


we find that

$$
\mathscr{A}_{2}^{-1}[\sin (\xi x) ; t]=\sqrt{ }\left(\frac{1}{2} \pi\right) \xi t Y_{0}(\xi t) .
$$

The method used in Sections 3, 4 rests essentially on two integral identities which we shall now derive.

From the definition (2.1) we have

$$
\int_{0}^{\infty} K(\xi, x) \hat{f}_{1}(x) d x=\sqrt{ }\left(\frac{2}{\pi}\right) \int_{0}^{\infty} K(\xi, x) d x \int_{0}^{x} \frac{f(t) d t}{\sqrt{\left(x^{2}-t^{2}\right)}} .
$$

Reversing the order in which we perform the integrations we see that the double integral on the right may be written in the form

$$
\sqrt{\left(\frac{2}{\pi}\right)} \int_{0}^{\infty} f(t) d t \int_{t}^{\infty} \frac{K(\xi, x) d x}{\sqrt{\left(x^{2}-t^{2}\right)}}
$$

which shows that

$$
\int_{0}^{\infty} K(\xi, x) \hat{f}_{1}(x) d x=\int_{0}^{\infty} f(x) \mathscr{A}_{2}[K(\xi, t) ; t \rightarrow x] d x .
$$

In a similar way we can show that

$$
\int_{0}^{\infty} K(\check{\zeta}, x) \hat{f}_{2}(x) d x=\int_{0}^{\infty} f(x) \mathscr{A}_{1}[K(\xi, t) ; t \rightarrow x] d x .
$$

3. Inversion of the operators $\mathscr{H}_{0}$ and $\mathscr{H}_{1}$. If we denote the operator of the Fourier sine transform by $\mathscr{F}_{3}$, so that

$$
\mathscr{F}_{s}[f(x) ; \xi]=\int\left(\frac{2}{\pi}\right) \int_{0}^{\infty} f(x) \sin (\xi x) d x
$$

we see from equations (2.13) and (2.7) that

$$
\mathscr{F}_{s}\left[\hat{f}_{1}(x) ; \xi\right]=\int_{0}^{\infty} f(x) J_{0}(\xi x) d x .
$$

We can write this equation in the alternative form

$$
\mathscr{F}_{s}\left[\mathscr{A}_{1}\{t f(t) ; x\} ; \xi\right]=\bar{f}_{0}(\xi),
$$

where $\bar{f}_{0}(\xi)$ is the Hankel transform of order zero of the function $f(x)$, defined by (1.l) with $v=0$.

Applying the operator $\mathscr{F}_{s}^{-1} \equiv \mathscr{F}_{s}$ to both sides of equation (3.1) we find that

$$
\mathscr{A}_{1}[t f(t) ; x]=\sqrt{ }\left(\frac{2}{\pi}\right) \int_{0}^{\infty} f_{0}(\xi) \sin (\xi x) d \xi
$$


and then, applying $\mathscr{A}_{1}^{-1}$ to both sides of this equation, that

$$
t f(t)=\sqrt{ }\left(\frac{2}{\pi}\right) \int_{0}^{\infty} f_{0}(\xi) \mathscr{A}_{1}^{-1}[\sin (\xi x) ; t] d \xi .
$$

Using equation (2.9) we see that this last equation is equivalent to the relation

$$
f(t)=\int_{0}^{\infty} \xi f_{0}(\xi) J_{0}(\xi t) d \xi .
$$

In other words: If $f_{0}(\xi)=\mathscr{H}_{0}[f(x) ; \xi]$, then $f(x)=\mathscr{H}_{0}\left[f_{0}(\xi) ; x\right]$, i.e. $\mathscr{H}_{0}^{-1}=\mathscr{H}_{0}$.

Similarly from equations (2.14) and (2.8) we see that

$$
\mathscr{F}_{s}\left[x \hat{f}_{2}(x) ; \xi\right]=\sqrt{ }\left(\frac{2}{\pi}\right) \int_{0}^{\infty} f(x) \mathscr{A}_{1}[t \sin (\xi t) ; x] d x=\int_{0}^{\infty} x f(x) J_{1}(\xi x) d x
$$

and hence that

$$
\mathscr{F}_{s}\left[x \hat{f}_{2}(x) ; \xi\right]=f_{1}(\xi)
$$

where $f_{1}(\xi)$ is the Hankel transform of order 1 of $f(x)$. Applying the operator $\mathscr{F}_{\text {a }}$ to both sides of this equation, we have

$$
x \hat{f}_{2}(x)=\mathscr{F}_{s}\left[f_{1}(\xi) ; x\right]
$$

which, in turn, we can write as

$$
f(t)=\mathscr{A}_{2}^{-1}\left[\frac{1}{x} \mathscr{F}_{s}\left\{f_{1}(\xi) ; x\right\} ; t\right]=\sqrt{ }\left(\frac{2}{\pi}\right) \int_{0}^{\infty} f_{1}(\xi) \mathscr{A}_{2}^{-1}\left[\frac{\sin (\xi x)}{x} ; t\right] d \xi .
$$

Using equation (2.10) we see that

i.e. that $\mathscr{H}_{1}^{-1}=\mathscr{H}_{1}$.

$$
f(t)=\mathscr{H}_{1}\left[f_{1}(\xi) ; t\right]
$$

4. Inversion of the operators $\mathscr{H}_{0}^{*}$ and $\mathscr{Y}_{0}$. From equations (2.14) and (2.11) we find that

$$
\mathscr{F}_{s}\left[\hat{f}_{2}(x) ; \xi\right]=\int_{0}^{\infty} f(x) \mathbf{H}_{0}(\xi x) d x,
$$

a result which can be written in the form

where

$$
\mathscr{F}_{s[}\left[\mathscr{A}_{2}\{t \vec{f}(t) ; x\} ; \xi\right]=f_{0}^{*}(\xi),
$$

$$
f_{0}^{*}(\xi)=\mathscr{H}_{0}^{*}[f(x) ; \xi] \equiv \int_{0}^{\infty} x f(x) \mathbf{H}_{0}(\xi x) d x .
$$

Applying the operators $\mathscr{F}_{3}$ and $\mathscr{A}_{2}^{-1}$ in turn to both sides of equation (4.1) we find that

$$
t f(t)=\sqrt{ }\left(\frac{2}{\pi}\right) \int_{0}^{\infty} f_{0}^{*}(\xi) \mathscr{A}_{2}^{-1}[\sin (\xi x) ; x \rightarrow t] d \xi
$$


Using equation (2.12) we see that

$$
f(t)=\int_{0}^{\infty} \xi f_{0}^{*}(\xi) Y_{0}(\xi t) d \xi
$$

Comparing equations (4.2) and (4.3) we find that

$$
\left(\mathscr{H}_{0}^{*}\right)^{-1}=\mathscr{Y}_{0} \text {. }
$$

\section{REFERENCES}

1. G. N. Watson, A treatise on the theory of Bessel functions, 2nd edition (Cambridge, 1944).

2. E. C. Titchmarsh, Introduction to the theory of Fourier integrals (Oxford, 1937).

\section{UNIVERSITY OF GLASGOW}

